\title{
Topological Phase Transition in Coupled Rock-Paper-Scissors Cycles
}

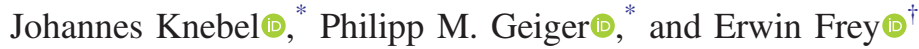 \\ Arnold-Sommerfeld-Center for Theoretical Physics and Center for NanoScience, \\ Department of Physics, Ludwig-Maximilians-Universität München, Theresienstrasse 37, D-80333 Munich, Germany
}

(Received 7 September 2020; revised 19 October 2020; accepted 5 November 2020; published 17 December 2020)

\begin{abstract}
A hallmark of topological phases is the occurrence of topologically protected modes at the system's boundary. Here, we find topological phases in the antisymmetric Lotka-Volterra equation (ALVE). The ALVE is a nonlinear dynamical system and describes, for example, the evolutionary dynamics of a rockpaper-scissors cycle. On a one-dimensional chain of rock-paper-scissor cycles, topological phases become manifest as robust polarization states. At the transition point between left and right polarization, solitary waves are observed. This topological phase transition lies in symmetry class $D$ within the "tenfold way" classification as also realized by $1 \mathrm{D}$ topological superconductors.
\end{abstract}

DOI: 10.1103/PhysRevLett.125.258301

Introduction.-Topological phases were discovered in condensed matter physics [1-5] and recently extended to classical physics such as topological mechanical metamaterials [6-10]. From a phenomenological point of view, topological phases are paramount for the following characteristics [4,5]. (i) Localization: Dynamical excitations become localized at the system's boundary. (ii) Robustness: These boundary modes are robust against perturbations of the system's parameters and noise. (iii) Phase transition: At the transition point between the topological phases, the dynamical mode expands throughout the whole system. From a theoretical point of view, topological phases are determined by nontrivial topological properties of the system's bulk, which are classified in terms of the system's symmetries. These properties give rise to gapless boundary modes and explain the observations (i)-(iii) $[4,5,9]$.

Thus far, the study of topological phases and their realization in soft-matter [11-14] and biological [15,16] systems has only started to develop, with a recent focus on active matter and fluid dynamics [17-20] and stochastic model systems [21-24]. As to what extent topological phases may determine the behavior of dynamical systems that arise, for example, in population dynamics was, however, not addressed. Ultimately, it would be interesting to design biological setups with nontrivial topological properties so that one obtains robust dynamical modes with the above characteristics (i)-(iii).

In this work, we make a step in this direction by showing that topological phases can be realized with the antisymmetric

Published by the American Physical Society under the terms of the Creative Commons Attribution 4.0 International license. Further distribution of this work must maintain attribution to the author(s) and the published article's title, journal citation, and DOI.
Lotka-Volterra equation (ALVE). The ALVE is a conceptually important model for studying coexistence in population dynamics $[25,26]$ and also describes the condensation of noninteracting bosons in driven-dissipative setups [27,28]. In population dynamics, the ALVE governs, for example, the evolutionary dynamics of the rock-paper-scissors (RPS) game, in which each of the three strategies dominates one strategy and is dominated by another one, such that all strategies coexist [29-32].

Mathematically speaking, the ALVE is a nonlinear, mass-conserving dynamical system defined on $S$ sites. The mass at each site $\alpha$ is denoted as $x_{\alpha}$ evolving according to the coupled ordinary differential equations:

$$
\frac{d}{d t} x_{\alpha}=x_{\alpha} \sum_{\beta=1}^{S} a_{\alpha \beta} x_{\beta}, \quad \alpha=1, \ldots, S .
$$

The real-valued $S \times S$ matrix $A=\left\{a_{\alpha \beta}\right\}_{\alpha, \beta}$ is antisymmetric $\left(a_{\alpha \beta}=-a_{\beta \alpha}\right)$ and defines how mass is transported between two sites in a nonlinear interaction $\sim x_{\alpha} x_{\beta}$.

Here, we study the ALVE on a one-dimensional chain of coupled rock-paper-scissors cycles ("RPS chain" see Fig. 1). We observe behaviors resembling key features of a topological phase transition: (i) mass polarizes to the right or left

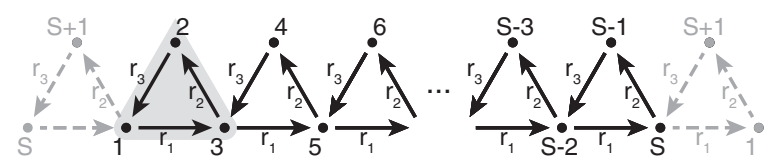

FIG. 1. One-dimensional chain of rock-paper-scissors cycles. The interactions on the $S$ sites of the RPS chain (one single RPS cycle highlighted) are captured by the antisymmetric matrix $A$ in Eq. (2). An arrow from one site to another indicates that mass is transported in this direction at a rate of $r_{1}, r_{2}, r_{3}>0$ following the ALVE (1); the skewness $r=r_{2} / r_{3}$ defines the control parameter. The auxiliary site $S+1$ facilitates periodic boundary conditions (dashed lines) within the framework of topological band theory. 


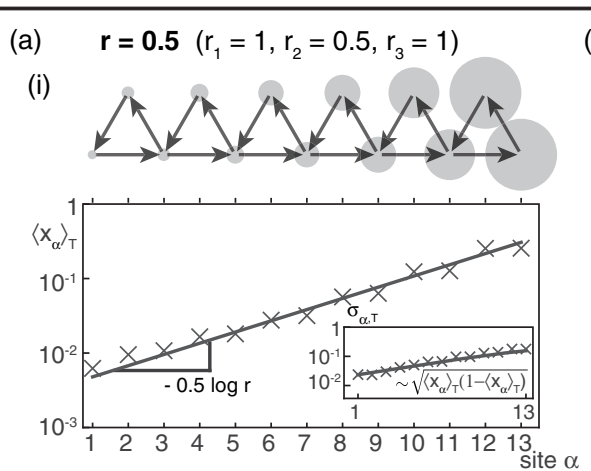

(ii)

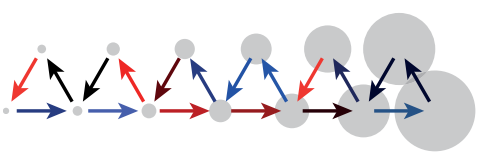

(b)

(i)
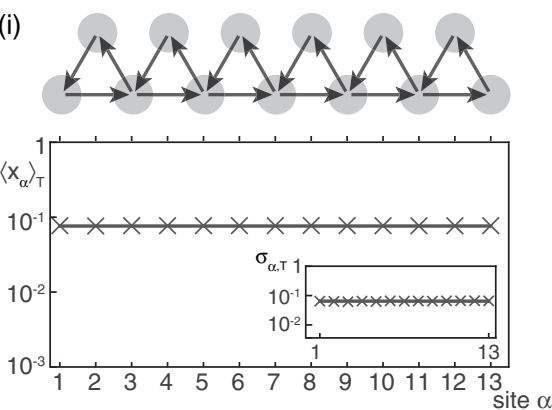

(ii)

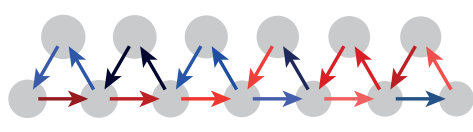

(c)
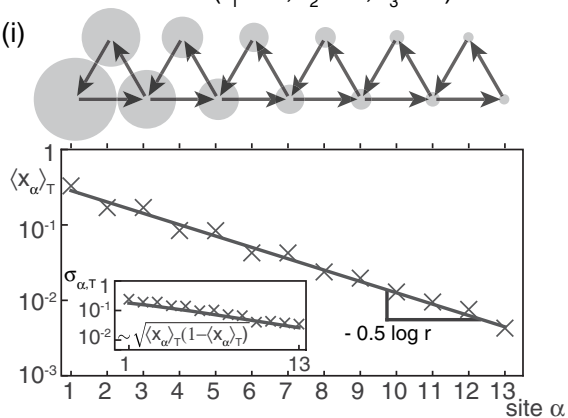

(ii)

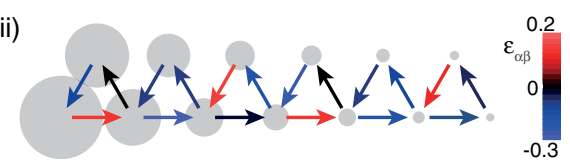

FIG. 2. Polarization of mass to the boundary. (a)-(c) (i) Temporal mass averages $\left\langle x_{\alpha}\right\rangle_{T}$ from single realizations of the ALVE (1) are depicted on the RPS chain (disk size encodes magnitude) and on lin-log scale for $T=5000$. Mass becomes polarized to the right for $r<1$ and to the left for $r>1$. For $r=1$, mass is uniformly distributed on the whole chain. Polarization is no state of rest (nonvanishing fluctuations $\sigma_{\alpha, T}$ around the averages; insets). (a)-(c) (ii) Polarization is robust against perturbations ( $\epsilon_{\alpha \beta}$ uniformly sampled in $[-0.30,0.20])$ with the same qualitative characteristics as without perturbations.

boundary of the RPS chain independent of the initial conditions; (ii) polarization is robust against perturbations of the model parameters; (iii) at the transition between left and right polarization, the overall mass expands on the whole chain and, moreover, solitary waves are observed. To explain these dynamics, we relate polarization of the ALVE (1) to properties of the antisymmetric matrix $A$. We show that the RPS chain encompasses a gap in the spectrum of $A$ and an intrinsic "particle-hole symmetry" and, thus, falls into the symmetry class $D$ within the tenfold way classification scheme of gapped free-fermion systems [5]. Hence, left and right polarization are distinguished by a $\mathbb{Z}_{2}$ invariant characterizing the topological phase transition. Intriguingly, the topological polarization states are entirely nonlinear in that they cannot be rationalized within the framework of linear wave theory. We envision that the described mechanism might guide one path to design topological phases in nonlinear dynamical systems accessible to biological experiments.

Model.-The RPS chain is composed of RPS cycles coupled in one dimension (Fig. 1) and represented by the antisymmetric matrix of size $S=2 n-1(n>0)$,

$A=\left(\begin{array}{ccccccccc}0 & r_{3} & -r_{1} & 0 & 0 & \cdots & 0 & 0 & 0 \\ -r_{3} & 0 & r_{2} & 0 & 0 & \cdots & 0 & 0 & 0 \\ r_{1} & -r_{2} & 0 & r_{3} & -r_{1} & \cdots & 0 & 0 & 0 \\ 0 & 0 & -r_{3} & 0 & r_{2} & \cdots & 0 & 0 & 0 \\ 0 & 0 & r_{1} & -r_{2} & 0 & \cdots & 0 & 0 & 0 \\ \vdots & \vdots & \vdots & \vdots & \vdots & \ddots & \vdots & \vdots & \vdots \\ 0 & 0 & 0 & 0 & 0 & \cdots & 0 & r_{3} & -r_{1} \\ 0 & 0 & 0 & 0 & 0 & \cdots & -r_{3} & 0 & r_{2} \\ 0 & 0 & 0 & 0 & 0 & \cdots & r_{1} & -r_{2} & 0\end{array}\right)$

with rate constants $r_{1}, r_{2}, r_{3}>0$. In our numerical simulations of the ALVE (1), time is rescaled such that $r_{1}=1$.
The ratio $r:=r_{2} / r_{3}$ serves as the control parameter for the dynamics and is referred to as skewness. The RPS chain can be thought of as a one-dimensional chain of nonlinear oscillators because each isolated RPS cycle represents a local oscillator in which mass oscillates between the different sites. For $r \neq 1$, mass is skewed towards certain sites within a single oscillating RPS cycle.

The initial masses in the ALVE (1) are normalized $\left(\sum_{\alpha} x_{0, \alpha}=1\right)$ and strictly positive $\left(x_{0, \alpha}>0\right.$ for all $\alpha$ such that the chain does not become disconnected or shortened). Because of the antisymmetry of $A$, the total mass is conserved for all times $t \geq 0 \quad\left(\sum_{\alpha} \partial_{t} x_{\alpha}=0\right)$. Furthermore, all masses remain bounded away from 0 $\left(x_{\alpha} \geq\right.$ const $>0$, for all $\alpha$ ) for all times for any choice of rates $r_{1}, r_{2}, r_{3}>0[28,33]$. In the context of population dynamics, this means that all strategies coexist.

Phenomenology.-In the numerical simulations of the RPS chain, we observed a surprisingly rich dynamics for how the mass is distributed depending on the skewness $r$.

(i) Localization: For skewness $r<1$, the overall mass on the RPS chain becomes polarized to the right boundary over time irrespective of the initial conditions [Fig. 2(a)(i)], whereas for $r>1$, mass polarizes to the left [Fig. 2(c)(i)] [34]. Polarization becomes manifest as an exponential decay of the average mass per site from the boundary into the bulk. We quantify this polarization by averaging the mass at every site over a time window $T \gg 1,\left\langle x_{\alpha}\right\rangle_{T}=1 / T \int_{0}^{T} d t x_{\alpha}(t)$. We observed that, for $r \neq 1$, average masses decay from the boundary into the bulk as $\left\langle x_{\alpha}\right\rangle_{T} \sim \exp \left(-\alpha / l_{p}\right)$ for $\alpha \geq 1$, numerically consistent with $l_{p}=2 / \ln r$ as the penetration depth. Such polarization arises for any initial mass distribution and is already visible for a small system size of $S=13$ (Fig. 2). 
Remarkably, the skewness $r=r_{2} / r_{3}$ alone determines whether the total mass polarizes to the left or right boundary of the chain. The values of all other parameters affect only the quantitative, but not the qualitative long-time behavior; see figures in the Supplemental Material [34].

The ALVE (1) is a deterministic dynamical system that approaches a stationary state at large times. This stationary state can be characterized by the average masses per site, $\left\langle x_{\alpha}\right\rangle_{T}$ (see above) and the fluctuations around the averages within a framework of thermodynamic equilibrium [26]. To quantify the fluctuations at site $\alpha$, we measured the standard deviation $\sigma_{\alpha, T}=\sqrt{\left\langle x_{\alpha}^{2}\right\rangle_{T}-\left\langle x_{\alpha}\right\rangle_{T}^{2}}$ [Figs. 2(a)(i) and 2(c)(i)]. Interestingly, the standard deviation scales with the average masses themselves as $\sigma_{\alpha, T} \sim \sqrt{\left\langle x_{\alpha}\right\rangle_{T}\left(1-\left\langle x_{\alpha}\right\rangle_{T}\right)}$, while their precise amplitude is determined by the initial conditions [34]. Taken together, also the fluctuations in the polarization state scale universally in that they decay exponentially into the bulk from the boundary where the mass is localized.

(ii) Robustness: Polarization is robust against perturbations of the model parameters. For example, Figs. 2(a)(ii) and 2(c)(ii) illustrate the polarization of mass to the boundary when the positive matrix entries are perturbed as $a_{\alpha \beta}^{\prime}=a_{\alpha \beta}\left(1+\epsilon_{\alpha \beta}\right)$; for $a_{\alpha \beta}<0$, the entry $a_{\alpha \beta}^{\prime}$ is obtained as $a_{\alpha \beta}^{\prime}=-a_{\beta \alpha}^{\prime}$ such that the perturbed matrix $A^{\prime}$ is antisymmetric. Here, all $\epsilon_{\alpha \beta}$ are independently drawn from the same uniform distribution (all realizations need to be greater than -1 to preserve the network topology of the RPS chain) [34]. As another example, we found that mass also becomes localized to the boundary when the coupling between even sites is introduced, that is, the links between sites $2 m$ and $2(m-1)$ for $m=2, \ldots, n-1$ are added to the RPS chain. Even with these additional couplings does the dynamics proceed into the same qualitative polarization states as without perturbation [34].

(iii) Phase transition: For $r=1$, the average masses expand throughout the whole chain, marking the transition point between the two polarization states. In the stationary state, the overall mass is on average uniformly distributed on the chain with $\left\langle x_{\alpha}\right\rangle_{T}=1 / S$ for all $\alpha(T \gg 1)$ : More generally, from our numerical simulations it turns out that it is not possible to tune any set of rate constants on the RPS chain such that one passes from polarization at one boundary to polarization at the other boundary without crossing a transition point at which the average masses expand throughout the whole chain.

Furthermore, we observed solitary mass waves at the transition point $r=1$. Mass packages that are suitably initialized at a few neighboring sites show solitonlike properties (Fig. 3): They are spatially confined; their shape does not change; and after an interaction with other solitary waves, their shape and speed remains unchanged [36,37]. It will be interesting to further characterize these solitary waves and connect them to already known solitons in similar setups [38-43].

Analysis.-Taken together, the combination of the observations of (i) localization, (ii) robustness, and (iii) phase transition share characteristic features of a topological phase transition underlying the behavior of the ALVE (1) on the RPS chain. In the following, we make this hypothesis rigorous. First, we outline how fixed points $\mathbf{x}^{*}$ of the ALVE $\left(\left.\partial_{t} \mathbf{x}\right|_{\mathbf{x}^{*}}=0\right)$ are determined by strictly positive kernel vectors of $A$. Second, we derive the qualitative changes in the dynamics from the bulk properties of $A$ within the framework of topological band theory. Third, we explicitly compute the kernel vector of $A$ and thereby confirm the results obtained from the topological band theory approach.

Fixed points.-For the RPS chain, there exists a unique, strictly positive kernel vector c for every choice of parameters $\left(A \mathbf{c}=\mathbf{0}\right.$ with $c_{\alpha}>0$ for all $\alpha$ and $\left.\sum_{\alpha} c_{\alpha}=1\right)$. This vector gives rise to the fixed point $\mathbf{x}^{*}=\mathbf{c}$ of the ALVE (1), and no further fixed points with $x_{\alpha}^{*}>0$ for all $\alpha$ exist [34]. The existence of a unique vector c derives from the cyclic structure of the concatenated RPS cycles; the explicit form of $\mathbf{c}$ is given below in Eq. (4). If the dynamics are initialized away from this fixed point $\mathbf{x}_{0} \neq \mathbf{c}$, the masses $\mathbf{x}$ do not approach the fixed point, but instead are confined on trajectories around the value of $\mathbf{c}$ because the quantity $E=x_{1}^{c_{1}} \ldots x_{S}^{c_{S}}$ is conserved. For such cases, it was shown that the average masses as introduced in the phenomenology section are given by the value of the fixed point $\left\langle x_{\alpha}\right\rangle_{\infty}=c_{\alpha}$ for all initial conditions [28,29]. This way, the long-time dynamics on the RPS chain (average masses $\langle\mathbf{x}\rangle_{\infty}$ ) are determined by algebraic properties of $A$ (kernel vector $\mathbf{c})$.

Topological band theory.-To further characterize the algebraic properties of $A$, the RPS chain is extended by the
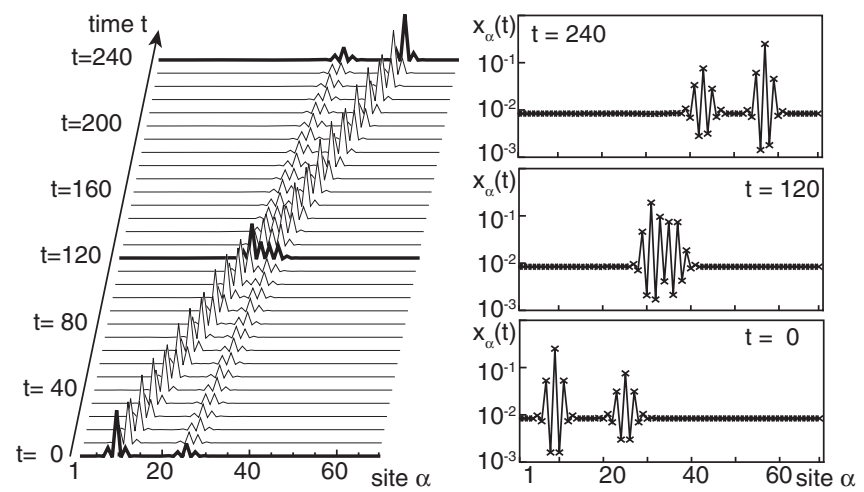

FIG. 3. Solitary waves at the transition point $r=1$. The suitably prepared wave package $(t=0)$ remains localized and travels along the RPS chain $(S=69)$ without changing its shape. In an interaction with another solitary wave $(t=120)$, the shapes of both wave packages remain unchanged afterwards $(t=240)$. The initial wave packages were numerically obtained from the dispersion of a mass peak at a single site. 
auxiliary site $S+1$ and periodic boundary conditions (PBC) are employed (Fig. 1). The corresponding antisymmetric matrix $A_{\mathrm{PBC}}$ is of size $2 n(n>0)$ and block circulant (that is, translationally invariant) $[44,45]$. To relate our approach to condensed matter physics, we define the "RPS Hamiltonian" $H:=i A_{\mathrm{PBC}}(i$ denotes the imaginary unit), which is a Hermitian matrix $\left(H^{\dagger}=H\right)$ with only real eigenvalues. Below, we analyze $H$ in the framework of topological band theory [46].

The spectrum of $H$ is characterized by its band structure. Starting from the eigenvalue equation for $H$ and exploiting translational invariance, the eigenvectors of $H$ can be decomposed into a plane wave part and a within-cell alignment part $\tilde{\mathbf{u}}(k)$, which fulfills the much simpler eigenvalue equation $\lambda(k) \tilde{\mathbf{u}}(k)=\tilde{H}(k) \tilde{\mathbf{u}}(k)$ for the Fourier-transformed Hamiltonian $\tilde{H} ; k$ denotes the wave number in the Brillouin zone $(k=(2 \pi / n) l$ and $l=$ $-\lfloor n / 2\rfloor, \ldots, 0,1, \ldots,\lfloor n / 2\rfloor-1)$. For the RPS chain, one finds

$\tilde{H}=\left(\begin{array}{cc}2 r_{1} \sin k & -r_{2} \sin k+i\left(r_{3}-r_{2} \cos k\right) \\ -r_{2} \sin k-i\left(r_{3}-r_{2} \cos k\right) & 0\end{array}\right)$,

which can be written as $\tilde{H}(k)=\mathbf{h}(k) \cdot \sigma$, with $\mathbf{h}(k)=$ $\left(h_{0}(k), h_{1}(k), h_{2}(k), h_{3}(k)\right)=\left(r_{1} \sin k,-r_{2} \sin k,-r_{3}+r_{2} \cos k\right.$, $\left.r_{1} \sin k\right) ; \sigma=\left(\sigma_{0}, \sigma_{1}, \sigma_{2}, \sigma_{3}\right)$ denotes the Pauli matrices [47] with $\sigma_{0}$ as the $2 \times 2$ identity matrix [34].

How the spectral and topological properties of $H$ depend on the control parameter $r$ can be derived from Eq. (3); see Fig. 4. First, the spectrum of $H$ exhibits two bands of eigenvalues, $\lambda^{+}$and $\lambda^{-}$, on the Brillouin zone $k \in[-\pi, \pi)$ since the unit cell is constituted of two sites $(2 m \rightarrow 2 m-1)$. For $r \neq 1$, the two bands are separated by a spectral gap that closes only for $r=1$ at $k=0$.

Second, the spectrum of $H$ is point symmetric with respect to the origin, $\lambda^{+}(k)=-\lambda^{-}(-k)$. This property follows from the intrinsic "particle-hole symmetry" of $H$ because it is defined by a real-valued antisymmetric matrix. In formal terms, $H$ fulfills the operator identity $\mathcal{C} \tilde{H}(k) \mathcal{C}^{-1}=-\tilde{H}(-k)$, with $\mathcal{C}:=\sigma_{0} \circ \kappa$ and $\kappa$ as the complex conjugation operator. However, $H$ does not have time-reversal or chiral symmetry. Thus, the RPS Hamiltonian $H$ falls into symmetry class $D$ in one dimension within the tenfold-way classification scheme of gapped free-fermion systems [5]. In fact, $H$ can be interpreted as a Bogoliubov-de Gennes mean-field description of superconductivity in the Majorana representation [5,48-50].

Gapped Hamiltonians in the symmetry class $D$ in 1D admit topological phases that are characterized by a $\mathbb{Z}_{2}$ invariant $[5,48,50]$. This invariant $\mathcal{M}$ is the sign of the Pfaffian of $A_{\mathrm{PBC}}$ and can be computed from $\tilde{H}$ as $\mathcal{M}=\operatorname{sign}\left(1-r^{2}\right)$ [50]. Thus, a topological phase transition occurs at $r=1$ : for $r<1$, the RPS Hamiltonian $H$ is in
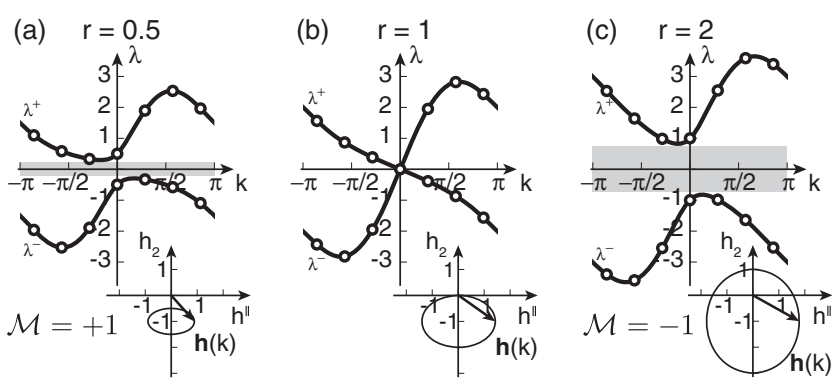

FIG. 4. Topological phase transition on the RPS chain. Pointsymmetric band structure of the RPS Hamiltonian (white dots denote eigenvalues of $H$ for $S=14$ ) with a spectral gap for $r \neq 1$ (gray shade). The band structures for $r<1$ and $r>1$ are topologically distinct in how the eigenvectors change within the Brillouin zone, which is quantified by the topological invariant $\mathcal{M}$ and visualized by whether $\mathbf{h}(k)$ winds around 0 [34].

the "topologically trivial phase" $(\mathcal{M}=+1)$; for $r>1$, the "topologically nontrivial phase" is attained $(\mathcal{M}=-1)$; see insets of Fig. 4 for an illustration. In other words, the two phases $(r<1$ and $r>1)$ are topologically distinct in that they cannot be smoothly deformed into one another without closing the spectral gap.

Through the so-called bulk-boundary correspondence $[5,6]$, topological properties of the bulk (periodic RPS chain with $S+1$ sites) become manifest at the boundary of the open system (open RPS chain with $S$ sites). More precisely, upon removing the auxiliary site $S+1$ and returning to the open RPS chain, the spectral gap for $r \neq 1$ is populated by a zero eigenvalue with a corresponding, topologically protected, strictly positive kernel vector, whose entries are localized at the boundary of the RPS chain [34]. This bulk-boundary correspondence is made rigorous by introducing a Toeplitz matrix as an intermediary between the two matrices $A$ and $A_{\mathrm{PBC}}$ and applying the Szegö-Widom theorem [51-53]. We conclude that the polarization states of the ALVE (1) correspond to gapless boundary modes. Thus, left and right polarization constitute topologically distinct stationary states, which cannot be transformed into each other without passing through the phase transition at $r=1$.

Kernel vectors.-Finally, we briefly present the exact form of the kernel vector $\mathbf{c}$ of $A$. To determine $\mathbf{c}$, we employed the graph-theoretical interpretation of the Pfaffian, such that the kernel of $A$ is related to the network representation of $A$ in Fig. 1; see $[33,34]$. As a result, the kernel vector $\mathbf{c}=\left(c_{1}, \ldots, c_{2 n-1}\right)$ can be written as

$$
\left(\begin{array}{c}
c_{2 m-1} \\
c_{2 m}
\end{array}\right)=\frac{1}{C}\left(\begin{array}{c}
r_{2} \\
r_{1}
\end{array}\right) r^{-(m-1)}, \quad m=1, \ldots, n-1,
$$

and $c_{2 n-1}=r_{2} r^{-(n-1)} / C ; C$ denotes the normalization constant and ensures $\sum_{\alpha} c_{\alpha}=1$. Thus, average masses $\langle\mathbf{x}\rangle_{\infty}=\mathbf{c}$ decay as $c_{\alpha} \sim \exp \left(-\alpha / l_{p}\right)$ with penetration depth $l_{p}=2 / \ln r$ from the boundary (analogously for 
$r<1$ ); see Fig. 2. This explicit construction of the kernel vector agrees with the result obtained within the approach of topological band theory and, thus, confirms the topological nature of the transition at $r=1$.

Discussion.-In this work, we report a topological phase transition in the stationary state of the ALVE (1) on the RPS chain. Stationary states are linked to strictly positive kernel vectors of the defining antisymmetric matrix. These kernel vectors are topologically protected and give rise to robust polarization of mass. Notably, these topological phases are entirely nonlinear in that they cannot be understood as the superposition of linear waves.

We envision that the results of this work could be extended to specific higher dimensions. In 2D, the symmetry class $D$ of the tenfold way classification admits topological phases characterized by the Chern number, whereas in 3D no topological phase transition occurs [5]. In 2D, such a topological phase should be observable as a unidirectional flow of mass at the system's boundary. Possible lattices might be constructed as two-dimensional carpets of RPS cycles as extensions of the chain.

Beyond the observation of topological phases in the ALVE, one might generalize this approach to other dynamical systems in biological physics, whose attractors are nonlinear oscillators or limit cycles [54], and ecology [55]. Here, we employed the ALVE on RPS cycles as the constituting building blocks, but other local oscillators may serve equally well. By suitably coupling these oscillators in the spirit of this work, we believe that topological phases as robust dynamical modes in biological systems could be designed.

We thank Chase Broedersz, Isabella Graf for discussions at early stages of this project, and Anton Souslov for discussions. This research was supported by the Excellence Cluster ORIGINS, which is funded by the Deutsche Forschungsgemeinschaft (DFG, German Research Foundation) under Germany's Excellence Strategy-EXC-2094-390783311, and the funding initiative "What is life?" of the VolkswagenStiftung.

*These authors contributed equally to this work. †rey@lmu.de

[1] K. v. Klitzing, G. Dorda, and M. Pepper, Phys. Rev. Lett. 45, 494 (1980).

[2] D. J. Thouless, M. Kohmoto, M. P. Nightingale, and M. den Nijs, Phys. Rev. Lett. 49, 405 (1982).

[3] F. D. M. Haldane, Phys. Rev. Lett. 61, 2015 (1988).

[4] M. Z. Hasan and C. L. Kane, Rev. Mod. Phys. 82, 3045 (2010).

[5] C.-K. Chiu, J. C. Y. Teo, A. P. Schnyder, and S. Ryu, Rev. Mod. Phys. 88, 035005 (2016).

[6] C. L. Kane and T. C. Lubensky, Nat. Phys. 10, 39 (2014).
[7] J. Paulose, B. G. Chen, and V. Vitelli, Nat. Phys. 11, 153 (2015).

[8] L. M. Nash, D. Kleckner, A. Read, V. Vitelli, A. M. Turner, and W. T. M. Irvine, Proc. Natl. Acad. Sci. U.S.A. 112, 14495 (2015).

[9] R. Süsstrunk and S. D. Huber, Proc. Natl. Acad. Sci. U.S.A. 113, E4767 (2016).

[10] H. Kedia, A. Souslov, and D. Z. Rocklin, arXiv:2008.01914v3.

[11] P. Delplace, J. B. Marston, and A. Venaille, Science 358, 1075 (2017).

[12] D. Zhou, L. Zhang, and X. Mao, Phys. Rev. Lett. 120, 068003 (2018).

[13] R. P. Pedro, J. Paulose, A. Souslov, M. Dresselhaus, and V. Vitelli, Phys. Rev. Lett. 122, 118001 (2019).

[14] C. Scheibner, W. T. M. Irvine, and V. Vitelli, Phys. Rev. Lett. 125, 118001 (2020).

[15] E. Prodan and C. Prodan, Phys. Rev. Lett. 103, 248101 (2009).

[16] L. Yamauchi, T. Hayata, M. Uwamichi, T. Ozawa, and K. Kawaguchi, arXiv:2008.10852.

[17] A. Souslov, B. C. van Zuiden, D. Bartolo, and V. Vitelli, Nat. Phys. 13, 1091 (2017).

[18] S. Shankar, M. J. Bowick, and M. C. Marchetti, Phys. Rev. X 7, 031039 (2017).

[19] A. Souslov, K. Dasbiswas, M. Fruchart, S. Vaikuntanathan, and V. Vitelli, Phys. Rev. Lett. 122, 128001 (2019).

[20] K. Sone and Y. Ashida, Phys. Rev. Lett. 123, 205502 (2019).

[21] A. Murugan and S. Vaikuntanathan, Nat. Commun. 8, 13881 (2017).

[22] K. Dasbiswas, K. K. Mandadapu, and S. Vaikuntanathan, Proc. Natl. Acad. Sci. U.S.A. 115, E9031 (2018).

[23] T. Yoshida and Y. Hatsugai, arXiv:2007.08730.

[24] E. Tang, J. Agudo-Canalejo, and R. Golestanian, arXiv: 2010.02845.

[25] V. Volterra, Leçons sur la Théorie Mathématique de la Lutte pour la Vie (Gauthier-Villars, Paris, 1931).

[26] N. S. Goel, S. C. Maitra, and E. W. Montroll, Rev. Mod. Phys. 43, 231 (1971).

[27] D. Vorberg, W. Wustmann, R. Ketzmerick, and A. Eckardt, Phys. Rev. Lett. 111, 240405 (2013).

[28] J. Knebel, M. F. Weber, T. Krüger, and E. Frey, Nat. Commun. 6, 6977 (2015).

[29] J. Hofbauer and K. Sigmund, Evolutionary Games and Population Dynamics (Cambridge University Press, Cambridge, England, 1998).

[30] T. Reichenbach, M. Mobilia, and E. Frey, Phys. Rev. E 74, 051907 (2006).

[31] J. C. Claussen and A. Traulsen, Phys. Rev. Lett. 100, 058104 (2008).

[32] A. Szolnoki, M. Mobilia, L.-L. Jiang, B. Szczesny, A. M. Rucklidge, and M. Perc, J. R. Soc. Interface 11, 20140735 (2014).

[33] P. M. Geiger, J. Knebel, and E. Frey, Phys. Rev. E 98, 062316 (2018).

[34] See Supplemental Material and videos at http://link.aps.org/ supplemental/10.1103/PhysRevLett.125.258301 for details, which includes Ref. [35].

[35] J. Knebel, T. Krüger, M. F. Weber, and E. Frey, Phys. Rev. Lett. 110, 168106 (2013). 
[36] M. Remoissenet, Waves Called Solitons (Springer, New York, 1999), Vol. 3.

[37] M. A. Ablowitz and P. A. Clarkson, Solitons, Nonlinear Evolution Equations and Inverse Scattering (Cambridge University Press, Cambridge, England, 1991).

[38] V. E. Zakharov, S. L. Musher, and A. M. Rubenchik, Sov. Phys. JETP 19, 151 (1974), https://ui.adsabs.harvard.edu/ abs/1974JETPL..19..151Z/abstract.

[39] S. V. Manakov, Sov. Phys. JETP 40, 269 (1975), https://ui .adsabs.harvard.edu/abs/1975JETP...40..269M/abstract.

[40] Y. B. Suris, The Problem of Integrable Discretization, Progress in Mathematics (Birkhäuser, Basel, 2003).

[41] Z. Yan, Nonlinear Anal. Theory Methods Appl. 64, 1798 (2006).

[42] B. G. Chen, N. Upadhyaya, and V. Vitelli, Proc. Natl. Acad. Sci. U.S.A. 111, 13004 (2014).

[43] R. Chaunsali and G. Theocharis, Phys. Rev. B 100, 014302 (2019).

[44] P. J. Davis, Circulant Matrices, Chelsea Publishing Series (Chelsea, New York, 1994).
[45] R. M. Gray, Found. Trends Commun. Inf. Theory 2, 155 (2006).

[46] C. L. Kane, Topological Insulators (Elsevier, New York, 2013), Chap. 1.

[47] G. Arfken, Mathematical Methods for Physicists (Academic Press, New York, 1985), Vol. 3.

[48] A. Y. Kitaev, Phys. Usp. 44, 131 (2001).

[49] S. Ryu and Y. Hatsugai, Phys. Rev. Lett. 89, 077002 (2002).

[50] J. C. Budich and E. Ardonne, Phys. Rev. B 88, 075419 (2013).

[51] E. Basor, J. Dubail, T. Emig, and R. Santachiara, J. Stat. Phys. 174, 28 (2019).

[52] J. Dubail, R. Santachiara, and T. Emig, J. Stat. Mech. (2017) 033201.

[53] P. Deift, A. Its, and I. Krasovsky, Commun. Pure Appl. Math. 66, 1360 (2013).

[54] B. Novák and J. J. Tyson, Nat. Rev. Mol. Cell Biol. 9, 981 (2008).

[55] S. P. Hubbell, The Unified Neutral Theory of Biodiversity and Biogeography (Princeton University Press, Princeton, NJ, 2001). 